\title{
Low Mass Scalar Mesons and Related Topics
}

\author{
Teiji Kunihiro*) \\ Yukawa Institute for Theoretical Physics, Kyoto University, Kyoto 606-8502, Japan
}

\begin{abstract}
We give a brief survey on the physical significance of the low-mass scalar mesons in QCD, and also report on recent lattice studies on the sigma and kappa mesons. The importance to explore the in-medium properties of the hadrons is mentioned.
\end{abstract}

\section{$\S 1$. Introduction; physical significance of low-lying scalar mesons}

A new era of hadron spectroscopy may have begun, which was initiated by the announcement of the pentaquark baryon ${ }^{1)}$ and many other possible exotic hadrons with mass larger than $2 \mathrm{GeV}$ containing heavy quarks. ${ }^{2)}$ These experimental developments prompted the intensive theoretical studies of QCD dynamics with new as well as old ideas on the structure and dynamics of the hadrons, which include chiral dynamics, multi-quark states with diquark correlations or molecular states and hybrids. ${ }^{2)}$ It is to be noted that such a controversy on the structure of hadrons is also the case for the scalar mesons below $1 \mathrm{GeV}$; they are the $I=0$ and $J^{P C}=0^{++}$ meson, i.e., the $\sigma(400-600)^{3)}$ seen in the $\pi-\pi$ scattering and in decay products from heavy-quark systems $\left.{ }^{4)}, 5\right)$ and also the iso-doublet strange scalar meson, i.e., the $\kappa$ with mass $\left.\sim 800 \mathrm{MeV} .^{5}\right)$

The significance of the $\sigma$ in hadron physics may be summarized as follows: ${ }^{6)}$ (i) The $\sigma$ with a mass $400 \sim 600 \mathrm{MeV}$ is responsible for the intermediate range attraction in the nuclear force; without the $\sigma$ contribution, any nucleus can not be bound, nor possible our existence. (ii) The existence of the $\sigma$ resonance can account for the $\Delta I=$ $1 / 2$ enhancement in the decay process $\mathrm{K}^{0} \rightarrow 2 \pi$ in comparison with $\mathrm{K}^{+} \rightarrow \pi^{+} \pi^{-} .7$ ) (iii) The empirical value of the $\pi$-N sigma term $\Sigma_{\pi N}=\left(m_{u}+m_{d}\right) / 2 \cdot\langle N|\bar{q} q| N\rangle \sim$ 40-60 MeV may be a reflection of the properties of the $\sigma$ as a collective mode: In fact, an analysis ${ }^{6), 8)}$ with a chiral model which describes the $\sigma$ meson as a collective mode gives an enhancement of the scalar charge of the nucleon as $\langle N|\bar{u} u+\bar{d} d| N\rangle \sim 9$, which is almost sufficient for accounting the empirical value of the sigma term with $\left(m_{u}+m_{d}\right) / 2 \sim 5.5 \mathrm{MeV}$, in contrast with the naive quark model which would give $\langle N|\bar{u} u+\bar{d} d| N\rangle=3$. See Ref.6) for more detailed discussions on the significance of the $\sigma$ meson in hadron physics.

The basic idea underlying the present report is that the low-energy hadron physics may be regarded as a study of the nature of QCD vacuum. In other words, hadron physics is a condensed matter physics of the QCD vacuum: In this point of view, hadrons are elementary excitations on top of the non-perturbative vacuum, although QCD itself is written solely in terms of quark and gluon fields. Such a viewpoint on the vacuum in quantum field theories was introduced by Nambu. ${ }^{9}$ )

*) e-mail address: kunihiro@yukawa.kyot-u.ac.jp 
Thus one sees that the iso-scalar and scalar meson can have an important aspect as a Higgs boson of QCD: ${ }^{6)}$ The chiral transition is a phase transition of QCD vacuum with $\langle\bar{q} q\rangle$ being the order parameter, as clearly shown by the lattice simulations. ${ }^{10)}$ In fact, $\mathrm{Nambu}^{9)}$ showed that an isoscalar-scalar meson with $J^{P C}=0^{++}$, i.e., the $\sigma$ emerges as a quantum fluctuations of the chiral order parameter $\left\langle:(\bar{q} q)^{2}:\right\rangle$ with the mass $2 M_{q}$ as another collective mode as the pion does, where $M_{q}$ is the dynamically generated quark mass. This picture should remain valid for QCD and the NJL model in fact works rather well for describing some aspects of the low energy hadron dynamics related to chiral symmetry and its dynamical breaking. ${ }^{11)}$

If a phase transition is of second order or weak 1st order, there exists "soft" modes which decreases it mass when the system approach the critical point; the soft modes are actually fluctuations of the order parameter of the phase transition. For chiral transition, the relevant fluctuation is described by $\left\langle(: \bar{q} q:)^{2}\right\rangle$, which has the same quantum numbers as the $\sigma$-meson does, i.e., $\left(I=0, J^{P C}=0^{++}\right)$. Accordingly the $\sigma$ meson can become the soft mode of chiral transition at $T \neq 0$ and/or $\rho_{B} \neq 0:{ }^{11), 12)}$ $m_{\sigma} \rightarrow 0, \Gamma_{\sigma} \rightarrow 0$ The lattice calculation ${ }^{10)}$ of the generalized mass $m_{\sigma}^{\text {gen }}=\chi_{\sigma}^{-1 / 2}$ defined in terms of the scalar correlation function $\left\langle(\bar{q} q)^{2}\right\rangle$ shows that the above picture is valid. In this respect, it is to be noticed that the peak position of the correlation function or the fluctuation $\left\langle(\bar{q} q)^{2}\right\rangle$ is used to identify the critical temperature of the chiral transition $T_{c}^{\chi}$, which is known to coincide with the critical temperature for the deconfinement $T_{c}^{\text {dec }}$ given from the peak position of the fluctuation of the Polyakov loop $\langle L\rangle .{ }^{10)}$

\section{$\S 2$. Low-lying scalar mesons in lattice QCD}

As mentioned in the previous section, there are controversies on the nature of the low-lying scalar mesons. In the non-relativistic constituent quark model, $J^{P C}=0^{++}$ is realized as a ${ }^{3} \mathrm{P}_{0}$ state, which implies that the mass of the $\sigma$ should be in the 1.2-1.6 $\mathrm{GeV}$ region. Therefore some mechanism is needed to down the mass. The possible mechanisms so far proposed include: (1) The color magnetic interaction between the di-quarks as advocated by Jaffe; ${ }^{13)}$ according to this conjecture, the $\sigma$ and other low-mass scalar mesons are tetra-quark states. (2) The collectiveness of the scalar mode as the pseudoscalar mode; a superposition of $q \bar{q}$ states, which collectiveness is due to chiral symmetry. ${ }^{9)}$ (3) These scalar mesons may be simply a resonance states of the NG bosons as the unitarized chiral dynamics could account for the existence of them. ${ }^{14)}$

Facing these problems with the $\sigma$, it would be interesting to explore the possible existence and the nature of the low-lying scalar mesons in the first-principle

calculation of QCD. Alford and Jaffe ${ }^{15)}$ examined whether the diquark correlations in the $\sigma$ channel is significant and thereby tried to have a suggestion the $\sigma$ is a tetraquark state. They found in fact a large attraction for heavy quark systems. But it should be warned that their calculations do not include the disconnected diagrams, i.e., closed quark loops, which means that the state being calculated is not a genuine flavor-singlet state. The SCALAR collaboration ${ }^{16)}$ performed a full QCD calculation 
of the $\sigma$ meson using the hybrid Monte Carlo method, which incorporated explicitly the disconnected diagrams by the $Z_{2}$-noise method with the $8^{3} \times 16$ lattice: The Wilson fermion with three quark masses and plaquette gauge action are employed; see 16) for the details of the lattice setup. The full QCD calculation includes the $\mathrm{q}-\overline{\mathrm{q}}$ creation and annihilation processes into the pure gluon states in the intermediate states so that possible tetra-quark, glue-ball states and so on can be taken into account, in contrast to the quenched approximation. The results are summarized as follows: The better signal of the $\sigma$ propagator is obtained for smaller quark masses and the contribution from the disconnected diagrams dominate the propagators over the connected ones. The simulation shows the existence of a clear $\sigma$ resonance, especially for smaller quark masses, and the resulting $\sigma$ is almost degenerated with the $\rho$ meson for the smallest quark mass, although the $\sigma$ mass becomes much smaller than the $\rho$ mass when the naive chiral limit is taken. Wada et al ${ }^{17)}$ have recently performed a lattice calculation of the $\kappa$ meson mass in the quenched level with a large lattice to see whether the flavored scalar meson to which the disconnected diagrams do not contribute can have a small mass as $800 \mathrm{MeV}$ as obtained in experiment. The result is negative as anticipated, which may mean that the scalar mesons including the $\sigma$ and the $\kappa$ should have exotic structures which can not be described by the simple constituent quark model.

\section{§3. Summary and concluding remarks}

We have emphasized that the $\sigma$ meson and other low-lying scalar mesons are as mysterious as the $\Theta^{+}$and other candidates of the exotics with charm: Naive quark model is in trouble for explaining such a low-mass state in the ${ }^{3} P_{0}$ state; it may be a four-quark or $\pi-\pi$ resonance state with no internal quark structure. The $\sigma$ might be also a collective $\mathrm{q}-\overline{\mathrm{q}}$ state to be identified as the quantum fluctuation of the order parameter of the chiral transition as Nambu originally suggested. The existence of such a collective mode in the scalar channel can account for some phenomena in hadron physics which otherwise remain mysterious. In short, the understanding of the nature or the even (non-)existence of the low-lying scalar mesons, especially of the $\sigma$, is important for a deep understanding of the QCD vacuum as well as the QCD/hadron dynamics.

A full QCD lattice simulation suggests the existence of a low-lying sigma, though its physics content, i.e., a tetraquark, a hybrid with the glue ball or the $\mathrm{q}-\overline{\mathrm{q}}$ collective state, is obscure: The disconnected diagram gives the dominant contribution to the $\sigma$ propagator. The simulation shows that $m_{\pi}<m_{\sigma}<m_{\rho}$ in the chiral limit. A quenched Lattice calculation suggests that the $\kappa$ can not be a normal $\mathrm{q}-\overline{\mathrm{q}}$ state, either.

To identify the nature of the $\sigma$ meson, exploring the possible change of the spectral function in the scalar channel in the hot and/or dense medium would be interesting, especially to examine whether the $\sigma$ meson can be really identified with the quantum fluctuations of the chiral order parameter; ${ }^{11), 12), 18), 19)}$ a peculiar enhancement of the spectral function in the $\sigma$ channel in the lowering energy side may be observed along with a partial restoration of chiral symmetry in the medium. ${ }^{19)}$ 
Recently, possible $N_{c}$-dependence of the nature of the $\sigma$ meson has been noticed by some authors ${ }^{20)} \mathrm{T}$. Schaefer showed that at $N_{c}=3$ the low mass $\sigma$ exists which is described as a linear combination of $\mathrm{q}-\overline{\mathrm{q}}$ and $(\mathrm{q} \overline{\mathrm{q}})^{2}$. However, for larger $N_{c}, m_{\sigma}$ goes up and the $\sigma$ becomes mainly composed of $\mathrm{q}-\overline{\mathrm{q}}$. The same problem is examined by Pelaez but somewhat different conclusions are deduced. The fate of a hadron in the large $N_{c}$ limit might also tell whether the hadron is an ordinary hadron as a Feshbach resonance or an extraordinary hadron. ${ }^{21)}$

\section{Acknowledgements}

This report include the results obtained in the lattice calculation done by the SCALAR collaboration. I am grateful to all the members of the SCALAR collaboration for the collaboration. This work was supported by the Grant for Scientific Research (No.17540250) and by the Grant-in-Aid for the 21st Century COE "Center for Diversity and Universality in Physics" of Kyoto University.

\section{References}

1) T. Nakano et al. [LEPS Collaboration], Phys. Rev. Lett. 91 (2003) 012002.

2) For reviews, see, C. Quigg, PoS HEP2005 (2006) 400; E. S. Swanson, Phys. Rept. 429, (2006) 243.

3) Particle Data Group Collaboration, S. Eidlman et al., Phys. Lett. B592 (2004) 1.

4) For example, see Possible existence of the sigma-meson and its implications to hadron physics, KEK Proceedings 2000-4; E791 Collaboration, Phys. Rev. Lett. 86 (2001) 770.

5) D. B. Bugg, Phys. Lett. B572 (2003) 1; E791 Collaboration, Phys. Rev. Lett. 89 (2002) 121801; BES Collaboration, Phys. Lett. B633 (2006) 681.

6) Teiji Kunihiro, Prog. Theor. Physics. Suppl. 120 (1995), 75.

7) T. Morozumi, C. S. Lim and I. Sanda, Phys. Rev. Lett. 65 (1990), 404.

8) T. Kunihiro and T. Hatsuda, Phys. Lett. B240 (1990), 209; T. Hatsuda and T. Kunihiro, Nucl. Phys. B387 (1992), 715.

9) Y. Nambu, Phys. Rev. Lett. 4, 380 (1960); Y. Nambu and G. Jona-Lasinio, Phys. Rev. 122 (1961) 345; 124 (1961) 246.

10) F. Karsch, Lect. Notes in Phys. 583 (2002), 209.

11) T. Hatsuda and T. Kunihiro, Phys. Rep. 247 (1994) 221.

12) T. Hatsuda and T. Kunihiro, Prog. Theor. Phys. 74 (1985), 765; Phys. Rev. Lett. 55 (1985), 158; Phys. Lett. B185 (1987), 304; the proceedings of IPN Orsay Workshop on Chiral Fluctuations in Hadronic Matter, September 26- 28, 2001, Paris, France, nucl-th/0112027.

13) R. L. Jaffe, Phys. Rev.D15, 267, 281 (1977); see also D. Black et al Phys. Rev.D59 (1999) 074026; L. Maiani et al Phys. Rev. Lett. 93 (2004) 212002.

14) See, for instance, J. A. Oller, E. Oset and A. Ramos, Prog. Part. Nucl. Phys. 45, 157 (2000).

15) M. Alford and R. L. Jaffe, Nucl. Phys. B578 (2000) 367; see also K. F. Liu, these proceedings.

16) SCALAR Collaboration, Phys. Rev. D70 (2004) 034504.

17) H. Wada et al, hep-lat/0702023

18) T. Hatsuda, T. Kunihiro and H. Shimizu, Phys. Rev. Lett. 82 (1999), 2840; D. Jido et al, Phys. Rev.D63 (2001), 011901; K. Yokokawa et al, Phys. Rev. C66 (2002), 022201.

19) D. Jido, these proceedings and the references cited therein.

20) T. Schafer, Phys. Rev. D68, 114017 (2003); J. Pelaez, Phys. Rev. Lett. 92, 102001 (2004).

21) R. L. Jaffe, hep-ph/0701038 and these proceedings. 